\title{
Wheel wear analysis of motor and unpowered car of a high- speed train
}

Zhiwei Wang ${ }^{\mathrm{a}}$, Ruichen Wang ${ }^{\mathrm{b}}$, David Crosbee ${ }^{\mathrm{b}}$, Paul Allen ${ }^{\mathrm{b}}$, Yunguang $\mathrm{Ye}^{\mathrm{c}}$, Weihua Zhang ${ }^{\mathrm{a}}$

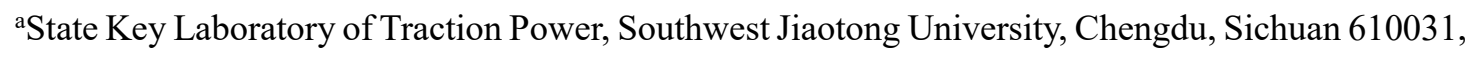
China;

${ }^{\mathrm{b}}$ Institute of railway research, University of Huddersfield, Huddersfield, HD1 3DH, UK.

${ }^{c}$ Institute of Land and Sea Transport Systems, Technical University of Berlin, Berlin, 10587, Germany

\begin{abstract}
The wheel-wear characteristics of the motor and unpowered car of a high-speed train were investigated, for the first time, by a wheel-wear prediction model comprising a wheel-wear submodel, an unpowered car dynamics sub-model, and a novel motor-car dynamics sub-model. The motor-car dynamics model considers the detailed structural characteristics and working mechanics of the traction transmission system. Assuming the real track parameters and track irregularities, it handles the nonlinear factors such as the traction characteristics, gear backlash, time-varying mesh stiffness, gear friction and wheel-rail contact. This study assessed the wheel-wear characteristics of a high-speed train by suitable performance indices (wheel-wear depth and contact patch energy). Finally, the model was validated by comparing the simulation results with those of field tests. The predicted and measured wheel wear were in good agreement. Both the wheel wear depth and contact energy were higher for the motor car than the unpowered car, because the traction torque on the wheelsets of the motor car increased the longitudinal creepage. During one re-profiling cycle, the transmission stability of the gear transmission system worsened with continuous wheel-wear. Furthermore, the proposed methods can assess the wheel wear and working status of the traction transmission system in the vehicle vibration environment of any rail vehicle.
\end{abstract}

\section{Keywords}

Wheel wear, traction transmission system, time-varying mesh stiffness, high-speed train, field experimental tests

* Corresponding author.

Email: R.Wang@hud.ac.uk 


\section{Introduction}

The use of high-speed trains in China has rapidly increased in recent years, due to the advantages of security, comfort and reduced journey times. However, the increasing speeds and operation mileages of fast trains intensifies the wheel-rail interaction, thereby worsening the service conditions of the vehicle system [1]. The wheel-rail interaction directly contributes to wheel wear, and dominates the vehicle running safety. The wear characteristics between the wheels and rails have attracted much attention by engineers and researchers [2-4].

Wheel-wear problems have been theoretically predicted and experimentally tested in the last two decades. The wheel wear is modelled by two approaches [5]. In the first approach, the removed material is supposed to be proportional to the dissipated energy within the contact patch and is handled by a single parameter $T_{\gamma}$ [6]. The second approach is the Archard's wear model, in which the material loss is proportional to the normal force and the sliding distance divided by the material hardness [7]. Braghin [8] proposed a mathematical model to predict wheel wear based on the wear index $T_{\gamma} / A$ and supported by the experimental tests. And then, Ignesti $[9,10]$ and Innocenti [11] make a lot of works to predict the wear of wheel and rail profile, and the later literature [11] further considered the complex railway lines using a statistic approach to get accurate results. In regard of the second approach, the Archard's model is widely used in wheel-rail wear prediction [12-14]. The literatures [15-17] compared different simulation approaches for wheel-wear prediction, and confirmed that the Archard's wear model implemented using FASTSIM is a suitable method for wheel-wear simulations. Bevan [18] developed a damage model that predicts the wheel-tread wear and rolling contact fatigue damage, which is validated by experimental data. In both modelling analysis and experimental test, Handa [19] showed that wheel wear is largely caused by the wheelrail tangential traction force. The wheel-rail tangential force is directly influenced by the traction/braking system and is explicitly coupled with the vehicle-track system [20-24]. Zhang et al. [25] investigated the wheel wear of a locomotive in a combined system of SIMPACK and Archard's wear model. At the same time, some research works investigated the influence of worn wheel profiles on the vehicle dynamics, and also pay much more attention on the vehicle and track parameters on the wheel wear $[24,26]$. However, the considerations of wheel wear on a realistic traction transmission system and the torsional vibration coupling effect on wheel wear, were not considered in the previous literature.

A typical high-speed train in China comprises motor cars and unpowered cars. The motor car includes traction transmission systems that transmit the motor torque to the wheelset, driving the vehicle forward. This study investigates the effects of the traction transmission system on the wheelrail interface. For this purpose, it establishes the multi-body system (MBS) vehicle dynamics of the motor and unpowered car in a new dynamics model based on dynamic vehicle and gear theory. To 
realistically capture the behaviour of the motor car, the model considers various nonlinear factors, such as the traction characteristics, time-varying mesh stiffness, fraction force of the gear pair and gear backlash, in detail. Next, the MBS vehicle model is incorporated into a wheel-wear prediction model. The dynamics model is combined with the wheel-wear assessment model to predict the wheel wear on the motor and unpowered cars. The wheel wear differences between the two car types, and their mechanism, are analysed in detail. The present work accounts for the torsional vibration of the traction transmission system and the traction characteristics of the motor, which significantly contribute to the wheel vibration. In addition, the performance of the gear transmission system is investigated during vehicle operation, which is meaningful for the design, condition monitoring and maintenance of this system. This model can potentially predict the wheel wear of the motor and unpowered car more realistically than previous approaches, and enables dynamic assessment of the gear transmission in the vehicle vibration environment.

\section{Modelling and simulation}

The vehicle-dynamics models of the motor and unpowered cars were developed in the SIMPACK environment, which simulates the wheel-rail interactions by Kalker's algorithm in FASTSIM, and hence calculates the wheel-rail contact forces. The traction transmission system is comprehensively built to reflect realistic traction conditions during vehicle operation. The dynamic responses, such as the creep velocities, contact area and normal force, are then obtained by the established dynamics model and input to the Archard wear model. The simulation procedure directly determines the wheel wear on the motor and unpowered cars.

\subsection{Multi-body system high-speed vehicle-dynamics model}

To study the wheel-wear evolution of the motor and unpowered cars in a high-speed train, the suspensions and interactions between the different components were determined in a detailed dynamic model based on the structural properties and working mechanism of the high-speed train. The motor car and unpowered car each comprised a rigid car body, rigid bogie frames, wheelsets, and four axle-boxes. The motor car additionally contained traction transmission systems for driving the vehicle forward. The motor-car dynamics model is based on a previously published model [22] with 31 rigid bodies and 86 degrees of freedom. In the proposed dynamics model, the kinematic constraints and suspension systems, such as the bump stop, spring stiffness, damper behaviour, timevarying mesh stiffness and the friction force of the gear pair, are implemented by nonlinear elements. The contact forces at the wheel-rail interface are simulated by the FASTSIM algorithm in the SIMPACK environment.

The traction transmission system consists of the traction motor, flexible coupling, a gear box, a gear 
pair and a wheelset, as shown in Figure 1. In the traction transmission system, the traction torque from the traction motor is transmitted to the pinion via flexible coupling, then delivered to the gearwheel via gear meshing. The resultant torque acts on the wheelset, exciting the forward-driving force at the wheel-rail interface. Because the traction transmission system and wheel-rail interface in the motor car are directly coupled by the gear meshing and wheel-rail interactions, the dynamics model of the gear pair must be prepared as accurately as possible. The high-speed motor car adopts a single-stage gear transmission system, and the parameters of the helical gear pair are those employed in the literature [17]. Based on the structural parameters, the gear pair can be established in the SIMPACK environment in detail. To capture the coupling effects between the transmission and vehicle systems, the vehicle-dynamics model accounts for the geometry, initial rotational angle, and rotational velocity. The helical gear-pair meshing effects during vehicle operation are determined by nonlinear factors such as the time-varying mesh stiffness, damping forces, friction forces and backlash. The model considers all geometric features of the gear tooth, and calculates the gear-pair responses online during the motor-car multi-body simulations. This calculation applies the contact modelling of the rigid gear wheels, which is implemented in SIMPACK [27]. The gear-pair model is pictured in Figure 1 (b). The dynamic responses of the gear transmission system, such as the circumferential force, dynamic transmission error and Hertzian pressure, were obtained by the proposed model to assess it performance evolution during a wheel re-profiling cycle of vehicle operation.

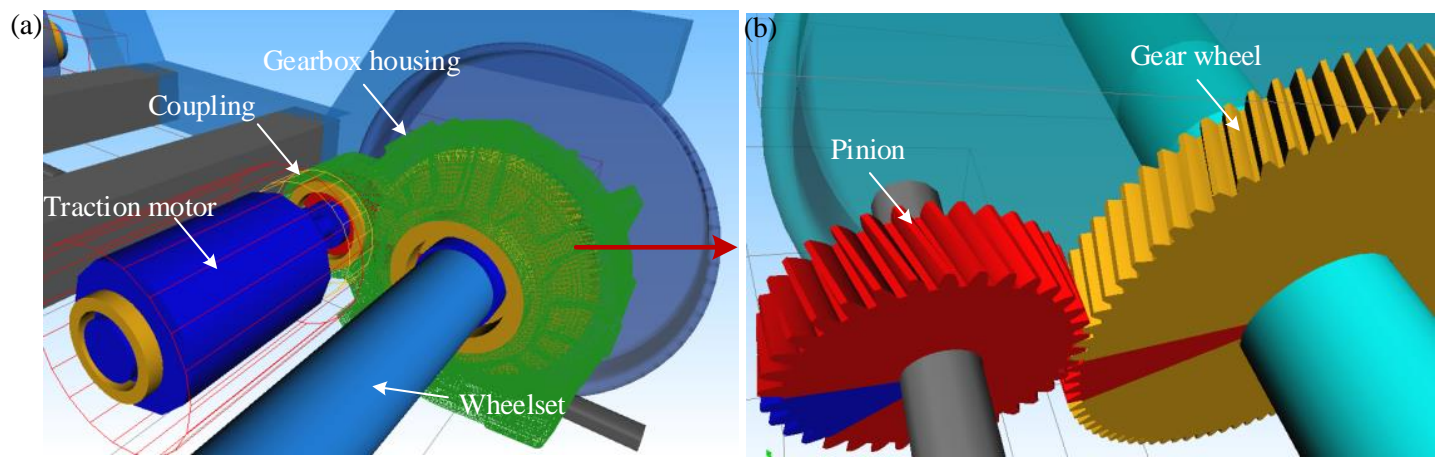

Figure 1 Developed model traction transmission system: (a) transmission system model and (b) gear-pair model.

\subsection{Wheel-wear model}

The wheel profile plays a dominant role in wheel-rail interactions. As such interactions affect the hunting stability, ride quality and other vehicle dynamics performances [28], predicting the wheel profile of a high-speed train during operation is highly important. In proposed model, the nonlinear Hertz contact theory is applied to perform the normal forces and, the state of the wheel-rail contact, such as the stick/slip status and creep parameters, is simulated by a FASTSIM algorithm and input 
to the wheel-wear model. The contact patch is assumed to be elliptical and the normal contact force is determined by Hertz theory [28]. The wheel wear, which is simulated by Archard's wear model $[7,12]$, is determined by the normal contact force, sliding distance and the hardness of the softer material using Eq. 1:

$$
V_{w}=K \frac{N D}{H},
$$

where $V_{w}$ is the wear volume, $D$ is the sliding distance, $N$ is the normal force, $H$ is the Vickers hardness of the soft material, and $K$ is the dimensionless wear coefficient. In previous work, the wear depth within an element of the contact patch was determined as

$$
\Delta Z=\frac{3 N K}{2 \pi a b H} \sqrt{1-\left(\frac{x}{a}\right)^{2}-\left(\frac{y}{b}\right)^{2}} \sqrt{s_{x}^{2}+s_{y}^{2}} \frac{\Delta x}{V_{c}},
$$

where $a$ and $b$ are the major and minor semiaxes of the ellipsoidal contact patch, respectively, $\Delta x$ is the element length in the forward running direction, $V_{c}$ is the velocity of a generic point relative to the contact patch, and $s_{x}$ and $s_{y}$ are the total slip velocities along the major and minor axes, respectively. The wear coefficients in Archard's model at different contact pressures and sliding velocities (see Figure 2) were investigated by Jendel and Berg [13]. The wear coefficients are divided into four regions. The most severe wear occurs in the top region, where the contact pressure exceeds 0.8 times the material hardness. The wear coefficient of the tread contact usually falls in the bottom left region, whereas that of the flange contact may fall into any of the four regions. The wear coefficients in Fig.2 are determined by experiential tests, which is an approximate one in the wheel-rail wear simulation [17]. The present simulation assumed the median values of the wear coefficients in the four regions and determined by the pressure and sliding velocity.

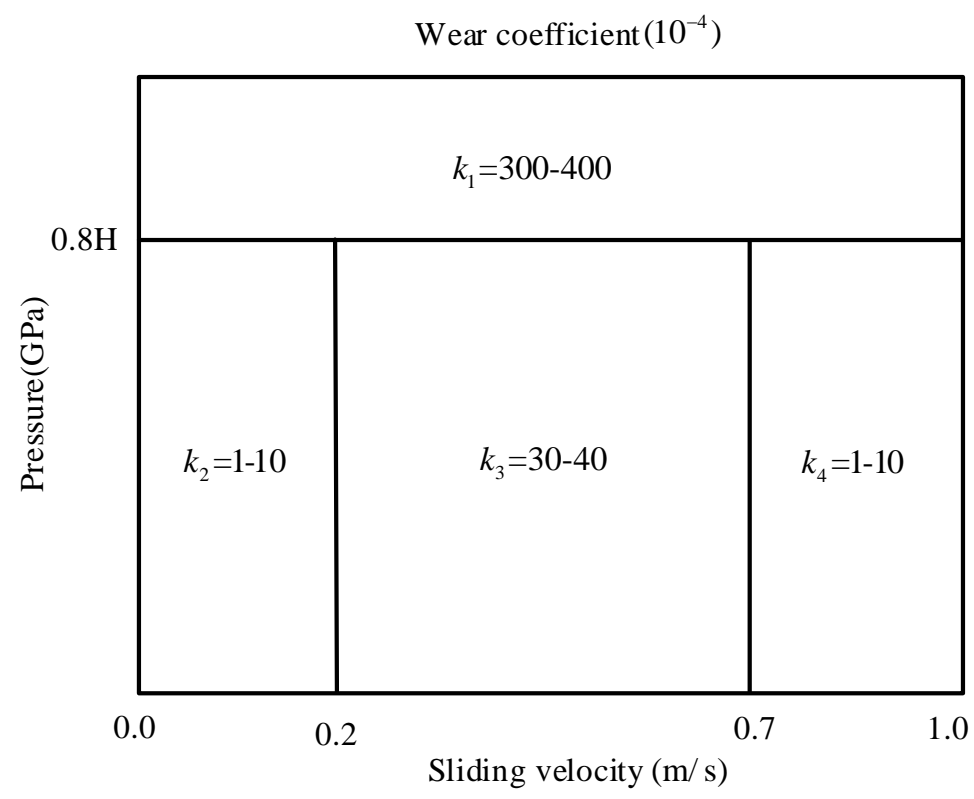

Figure 2 Wear coefficients in Archard's model. 


\subsection{Wheel wear prediction procedure}

A measured track for a main high-speed line of China was used for the simulation, these are listed in Table 1. In the wheel wear prediction procedure, the track is updating itself in each loop. According to the parameters in Table 1, there are six different type tracks with different radii, which make up different proportions of a 5-km unit length (expressed as percentages). Each track comprises the tangent track and curve track, and their length are set based on the percentages in Table 1. Besides, the tangent track and the curved tracks with different superelevations and transitions. Hence, there are six tracks with different parameters in the simulations. Besides, the same track irregularities are used for all cases. Therefore, apart from the rail irregularities, the wheel-rail interaction differs on each track due to different track settings. It can be noted that the percentages of the right and left curves are assumed identical throughout the simulation process.

Table 1 Track geometry properties of a high-speed railway line in China

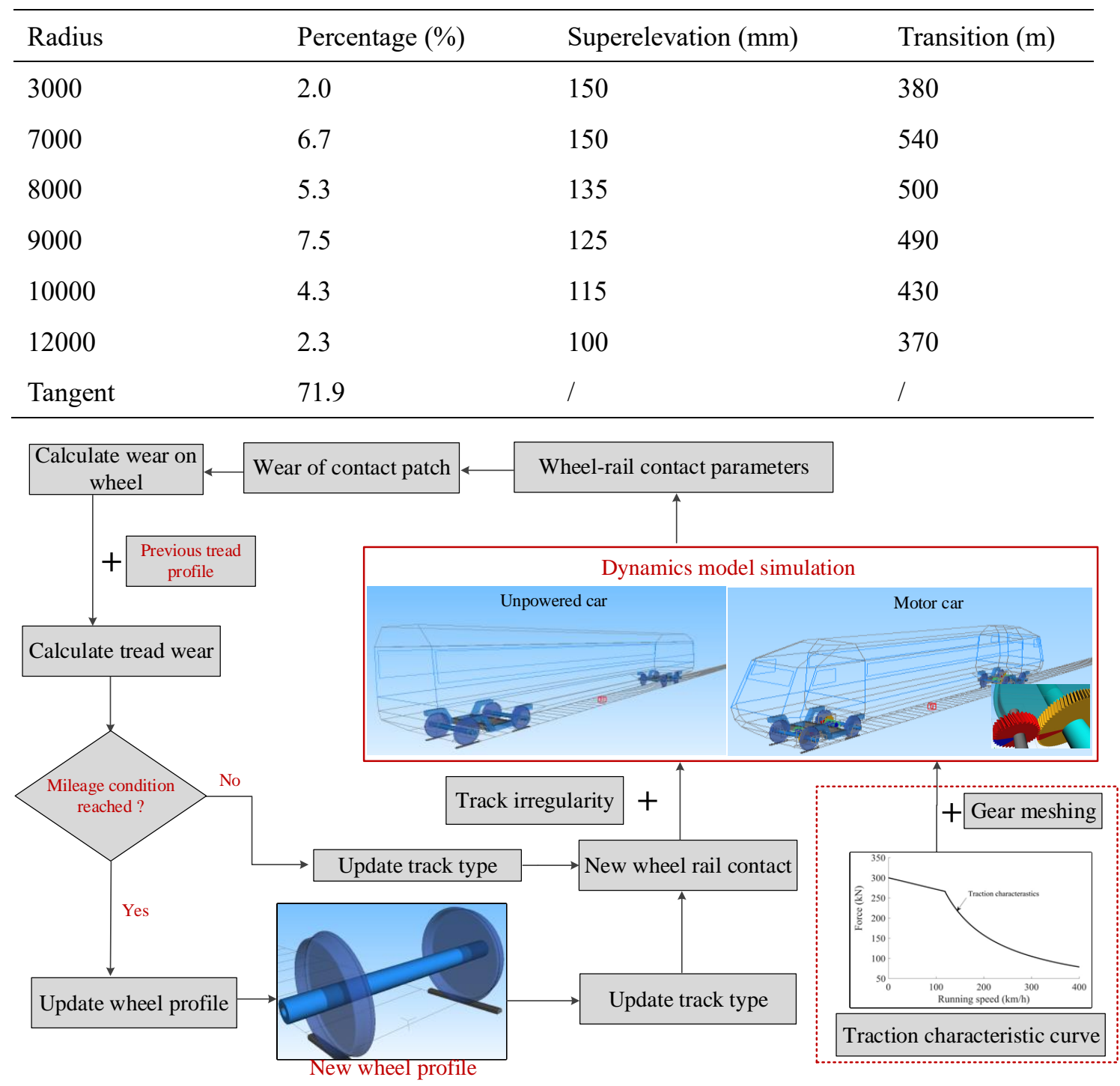

Figure 3 Wheel-wear prediction procedure.

Figure 3 is a flow chart of the main procedure in the wheel-wear prediction model. The dynamics 
model consists of three types of subsystems, namely, the MBS vehicle subsystems, the track subsystems, and the wheel-rail interface. The vehicle subsystems comprise two dynamic parts: the motor car and unpowered car, in which the vehicle dynamics are simulated. In each simulation interval, once the dynamic simulation is completed, the results of the wheel-rail interface are passed to the wheel-wear calculation, which calculates the tread wear and predicts the worn tread profile depends on the profile calculated by previous step. And then, the wheel profiles in the vehicle model are updated according to the distance travelled limitation $(1500 \mathrm{~km})$ before the next dynamic simulation. Once the distance limitation is reached, the wheel profile would be updated. The track is also updated successively from one of the six-track category. If the mileage limitation is not reached, the wheel profile keeps unchanged and the track type also be updated in the next simulation. Based on the newly regenerated wheel-rail interface, the dynamics model enters the next loop.

\section{Simulation analysis and discussion}

Unlike previous research, the proposed model considers the power transmission path from the traction motor to the wheelset via the traction transmission system, enabling a more realistic simulation of the motor acceleration process. The simulation adopts the real traction characteristics of the traction motor in an operating high-speed train, as shown in Figure 4. The acceleration process of the unpowered is consistent with that of the motor car. The numerical analysis is performed on the different types of track listed in Table 1. The wheel-wear characteristics of the motor and unpowered car are theoretically analysed below.

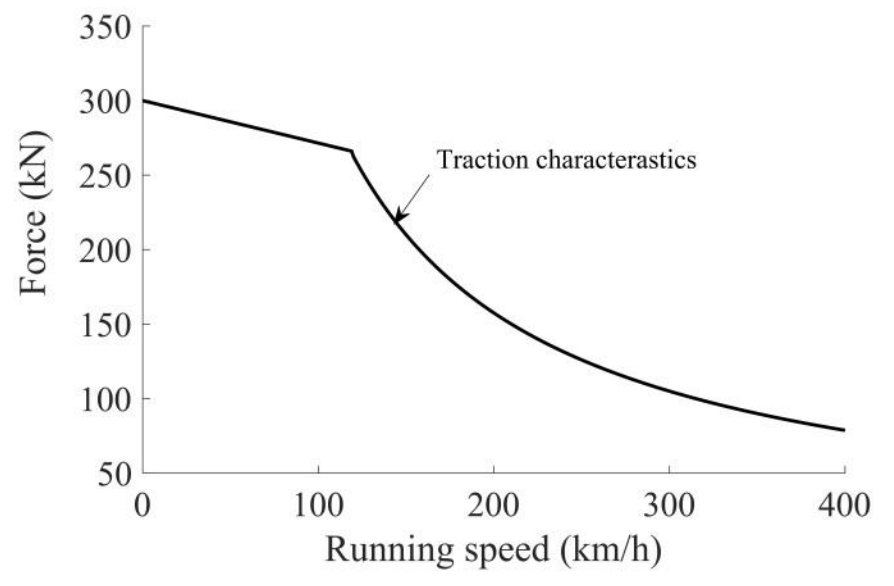

Figure 4 Tractive characteristic curve.

Additionally, the performance of the gear transmission system in terms of gear mesh force, dynamic transmission error (DTE) and gear contact stress is assessed in a wheelset re-profiling cycle. The DET of the one-stage gear system of high-speed train can be calculated by Eq.3 [20]:

$$
X_{D E T}=R_{p} \theta_{p}-R_{g} \theta_{g}-l_{1} \beta_{\text {gearb }}
$$


where $R_{p}$ and $R_{g}$ are the gear base radius of the pinion and the gear, respectively; $\theta_{p}$ and $\theta_{g}$ are the rotation angular of the pinion and gear, $l_{1}$ is the longitudinal distance between the centre of pinion and wheelset axle, $\beta_{\text {gearb }}$ is the rotational angular displacement of the gearbox housing.

\subsection{Predicted wheel wear analysis}

The effects of track curve radius were assessed using the initial wheel profile along each different curve radii. Figure 5 shows the wear depth of the wheel profile after an operating mileage of 3000 $\mathrm{km}$ on different tracks. The wear amount of the motor and unpowered cars varies considerably depended on the curve radius; specifically, the wheel-wear width decreased with increasing rail curve radius. This trend is attributed to the varying wheel-rail interactions. Moreover, the motor car clearly developed a deeper wheel wear than the unpowered car, at any track curve radius.
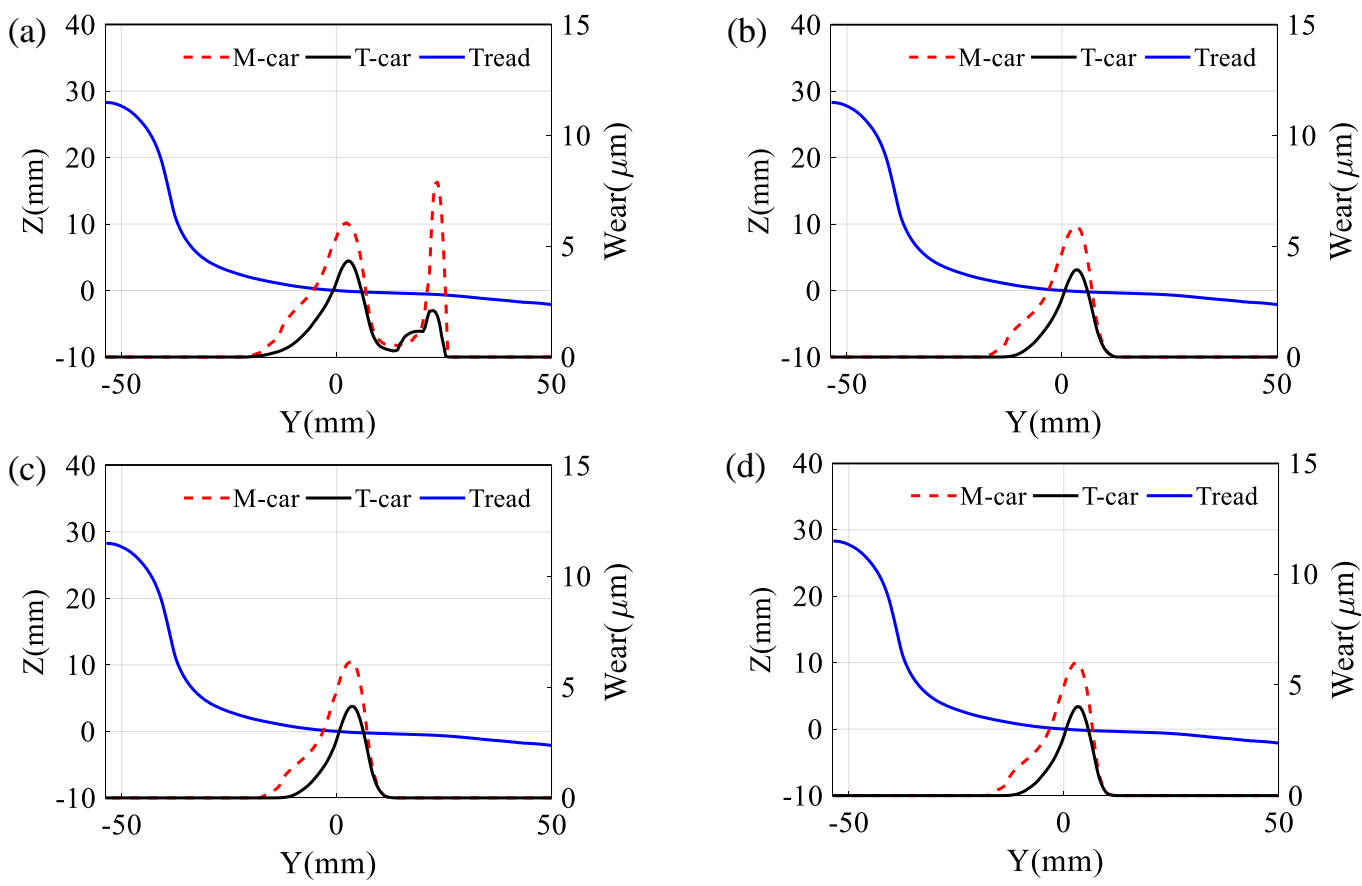

Figure 5 Wheel wear depths of a high-speed train after $3000 \mathrm{~km}$ operation on tracks with different track curve radii: (a) $3 \mathrm{~km}$, (b) $5 \mathrm{~km}$, (c) $9 \mathrm{~km}$ and (d) $12 \mathrm{~km}$.
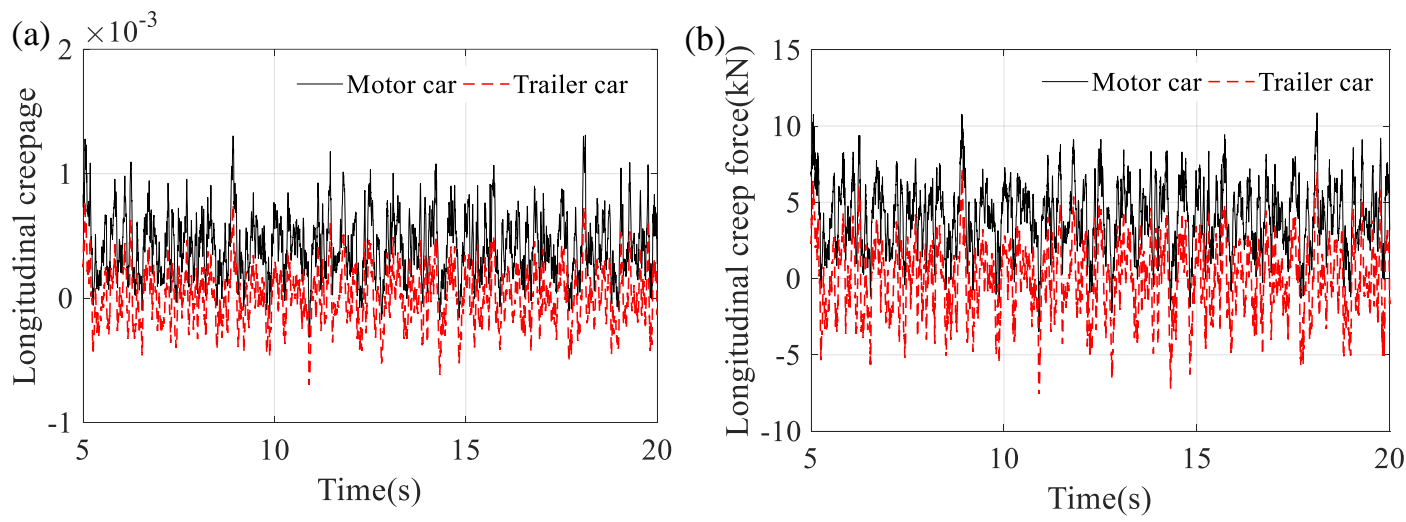

Figure 6 Comparison of wheel-rail interface responses of the motor and unpowered cars: (a) 
longitudinal creepage and (b) creep force.

Figure 6 shows the longitudinal creepage and longitudinal creep force of the motor and unpowered cars travelling at $300 \mathrm{~km} / \mathrm{h}$ along a straight track. The longitudinal creepage of the motor car was clearly larger than that of the unpowered car, because the motor car experiences a gear meshing force from the traction motor, which is transmitted by the traction transmission system. The wheelwear depth was proportional to the slip velocity, as expected from Eq. 2. The maximum amplitudes of the longitudinal creep force in the motor and unpowered car were $13.2 \mathrm{kN}$ and $9.2 \mathrm{kN}$, respectively. In addition, the mean longitudinal creep force per wheel of the motor car (required to counteract the running resistance) was $3.3 \mathrm{kN}$. However, as no extra forces acted on the wheelset of the unpowered car, the mean longitudinal creep force of each unpowered car wheel was nearly 0 $\mathrm{kN}$. According to Eq. 2, the higher longitudinal creepage contributes to the higher wheel-wear depth of the motor car than the unpowered car. This expectation is validated in the present simulation results.
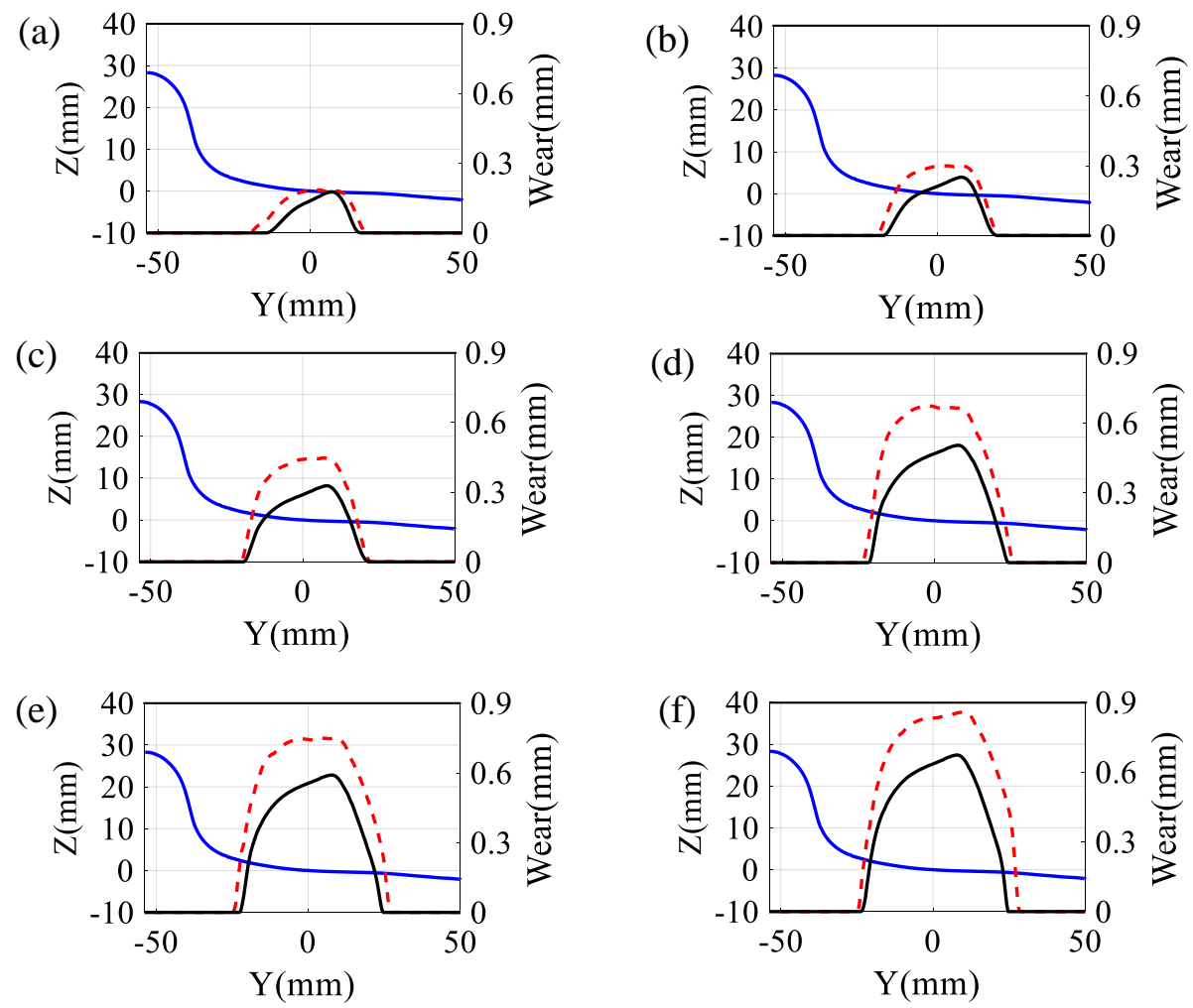

Figure 7 Tread wear-depths in the wheels of the motor car (red dashed curves) and unpowered car (black solid curves) after different operation mileages: (a) $3 \times 10^{4} \mathrm{~km}$, (b) $6 \times 10^{4} \mathrm{~km}$, (c) $9 \times 10^{4} \mathrm{~km}$, (d) $15 \times 10^{4} \mathrm{~km},(\mathrm{e}) 18 \times 10^{4} \mathrm{~km}$ and (f) $21 \times 10^{4} \mathrm{~km}$. The blue curves plot the wheel profile.

Figure 7 shows the wheel-wear depths of the motor and unpowered cars after different operating mileages. The wheel-wear depths of the motor and unpowered car were very similar at running distances up to $6 \times 10^{4} \mathrm{~km}$ (Figure 7 (a) and (b)). At larger distances $\left(\geq 9 \times 10^{4} \mathrm{~km}\right)$, the wheel-wear 
depth was clearly larger in the motor car than the unpowered car (Figure 7 (c)). It can be concluded that the wheel-wear depths of the motor and unpowered car diverge with increasing operating mileage. After $21 \times 10^{4} \mathrm{~km}$ (Figure 7 (f)), the maximum wheel-wear depth was $26.9 \%$ higher in the motor car than in the unpowered car $(0.85 \mathrm{~mm}$ versus $0.67 \mathrm{~mm})$. Moreover, although the motor car consists the traction transmission system, the calculation time of the dynamic responses and the wheel wear is slightly different for the power and unpowered cars in the demonstrated simulation cases.

\subsection{Contact patch energy}

The wheel wear can be assessed from the contact patch energy [29,30]. In this approach, the material loss is assumed to be proportional to the friction energy dissipated in contact patch $T_{\gamma}$, where $T_{\gamma}$ is given by

$$
T_{\gamma}=\left[\mathrm{T}_{y} \gamma_{y}\right]+\left[\mathrm{T}_{x} \gamma_{x}\right]+\left[M_{z} \omega_{z}\right]
$$

Here, $T_{y}$ and $T_{x}$ are the longitudinal and lateral creep force, respectively, and $M$ denotes the spin creep moment. $\gamma_{y}, \gamma_{x}$ and $\omega$ are the corresponding creepage in the directions of the longitudinal, lateral and spin.

Figure 8 displays the mean contact patch energy of the leading wheelset on motor and unpowered car for different curve radii and travel distances. The mean contact patch energy of both cars increased with travel distance. In addition, the contact patch energy of the vehicles was highest on the tightest curve (with radius $3 \mathrm{~km}$ ) after all travel distances. Under all simulations, the contact patch energy was higher in the motor car than in the unpowered car, meaning that the wheel-wear was more severe in the motor car than in the unpowered car.

(a)

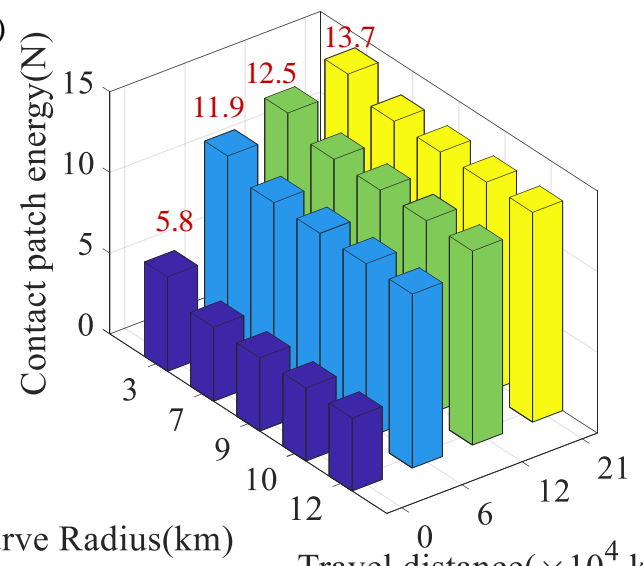

(b)

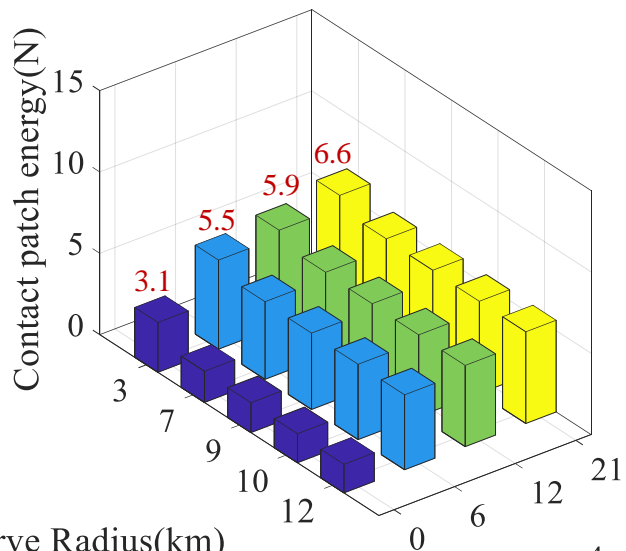

Travel distance $\left(\times 10^{4} \mathrm{~km}\right)$

Figure 8 Wheel contact energy after operating over different travel distances and curve radii: (a) motor car and (b) unpowered car. 


\subsection{Evaluation of gear transmission system}

As the key component of the motor car, the gear transmission system plays the role of delivering the power from traction motor to the wheelset to drive the car forward. Unlike previous dynamic models, the proposed model directly couples the vibration of the traction transmission system to the vehicle via gear meshing and the wheel-rail interaction. As the wheel-rail interaction influences the wheel-profile wear, the proposed dynamics model more realistically reflects the dynamic performance of the gear transmission system under the effects of wheel wear. To investigate the service status of the gear transmission system, its dynamic performance (gear mesh force, DTE and gear contact stress) was assessed throughout a re-profile cycle.

Figure 9 shows the dynamic performances of the gear transmission system over different operation mileages. The dynamic performance evolution of gear transmission system with the operating mileage was assessed by the standard deviations (stds) of the gear mesh force, the DET, and the contact stress, plotted as functions of mileage in Figure 9 (a), (b) and (c), respectively. The maximum amplitude of the contact stress versus mileage is plotted in Figure 9 (d). Although the track curve radius did not obviously affect the gear transmission system, all four indexes increased with increasing operation mileage throughout a reprofiling cycle. This trend indicates that continuous wheel wear degrades the transmission stability. After $21 \times 10^{4} \mathrm{~km}$ operation mileage, the stds of the gear mesh force, DTE, gear contact stress and maximum contact stress were 39.8\%, 31.6\%, 39.0\% and $10.1 \%$ higher, respectively, than their pre-operation values $(0 \mathrm{~km}$ mileage). Therefore, with increasing operation mileage, the degradation in the dynamic performance of the gear transmission system is attributable to increasing wheel wear. 
(a)

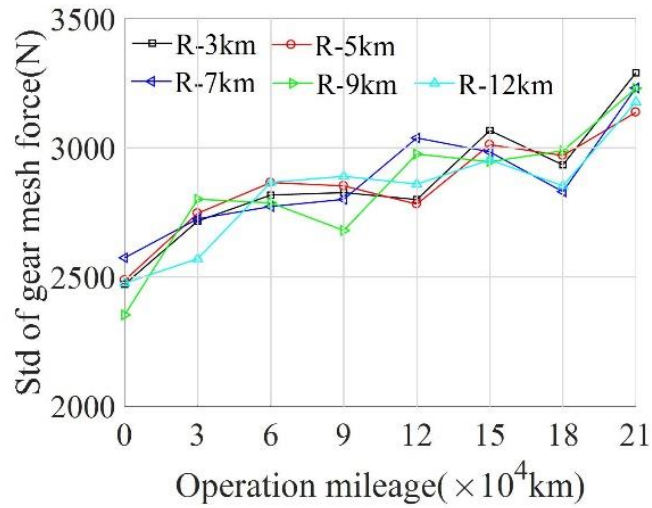

(c)

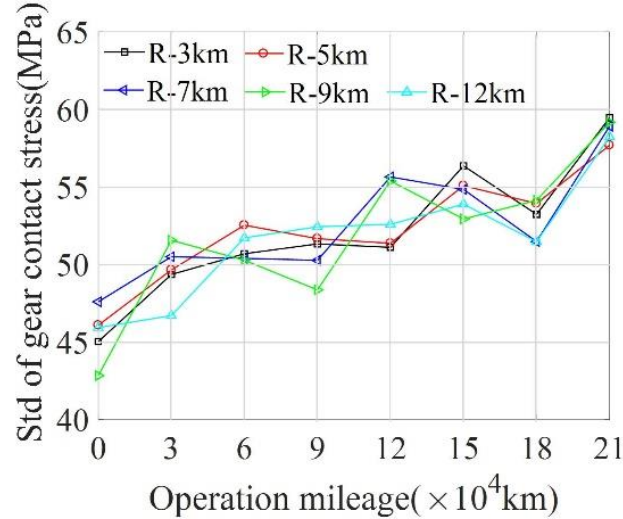

(b)

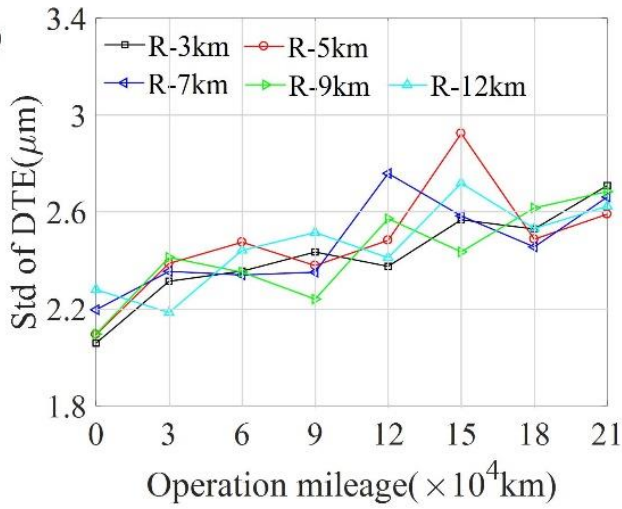

(d) 580

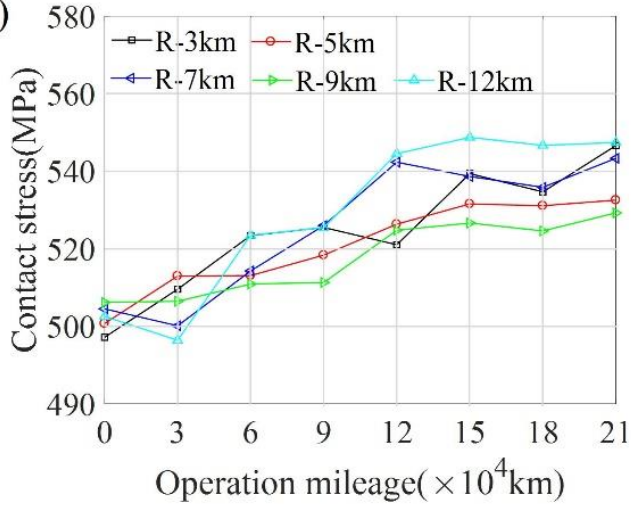

Figure 9 Dynamic responses of the gear transmission system over different operation mileages:

Standard deviations (stds) of (a) gear mesh force, (b) DTE, and (c) gear contact force. (d) the maximum amplitude of the gear contact stress.

\section{Experimental validation of the proposed model}

The proposed model was validated against long-term experimental tests. The test specimen was a typical high-speed train in China, which runs at a commercial running speed $300 \mathrm{~km} / \mathrm{h}$. Figure 10 illustrates the wheel tread profile measuring instrument, which is designed by Traction Power State Key Laboratory at the Southwest Jiaotong University. During the measurement process, the instrument is fixed at the wheel by the chuck, and the profile curves can be measured and recorded by the measuring arm. In order to reduce the test error, each wheel is tested at twelve different cross surfaces in a circle of the wheel. Next, the mean value of all the measured tread profiles applied to perform the wheel real profile curves. The repairing period of the wheels is set to approximately $210,000 \mathrm{~km}$ distance, and the wheel tread profiles are measured about 30,000 km each time. All wheel profiles used in power and unpowered cars of the selected high-speed train in a whole repairing circle are measured. The field measurements revealed flange wear imposed by the very small curves in the depot, which was not considered in the numerical simulation. However, as flange wear is minimal during usual operation on the main lines in a high-speed train network [28], the flange wear observed in the field tests was ignored. 


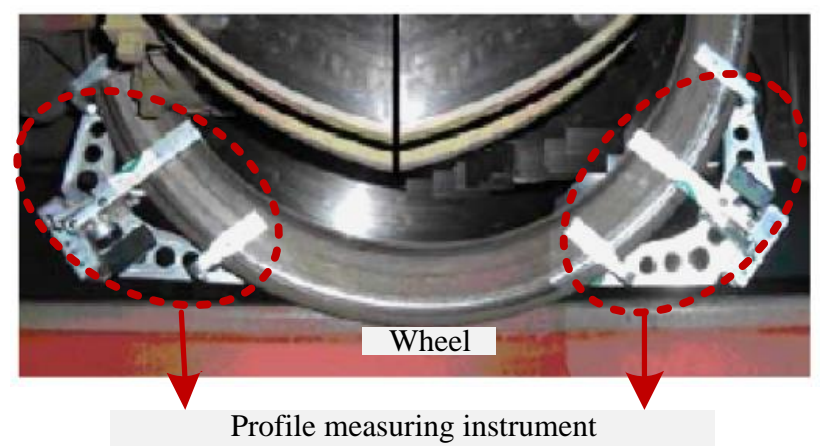

Figure 10 Experimental test of the wheel tread profile

Figure 11 compares the wear profiles of the motor and unpowered cars obtained in the field experiments and numerical simulations. Here the unpowered and motor cars are designated by ' $\mathrm{T}$ ' and ' $M$ ', respectively. The measured wear of both cars were wider in the experiments than in the simulation results, but the wear depths were consistent between the two results. The discrepancy can be explained by the track conditions over the whole line, which were assumed as representative in the model. In both experiments and simulations, the wheel-wear depth was higher in the motor car than in the unpowered car.
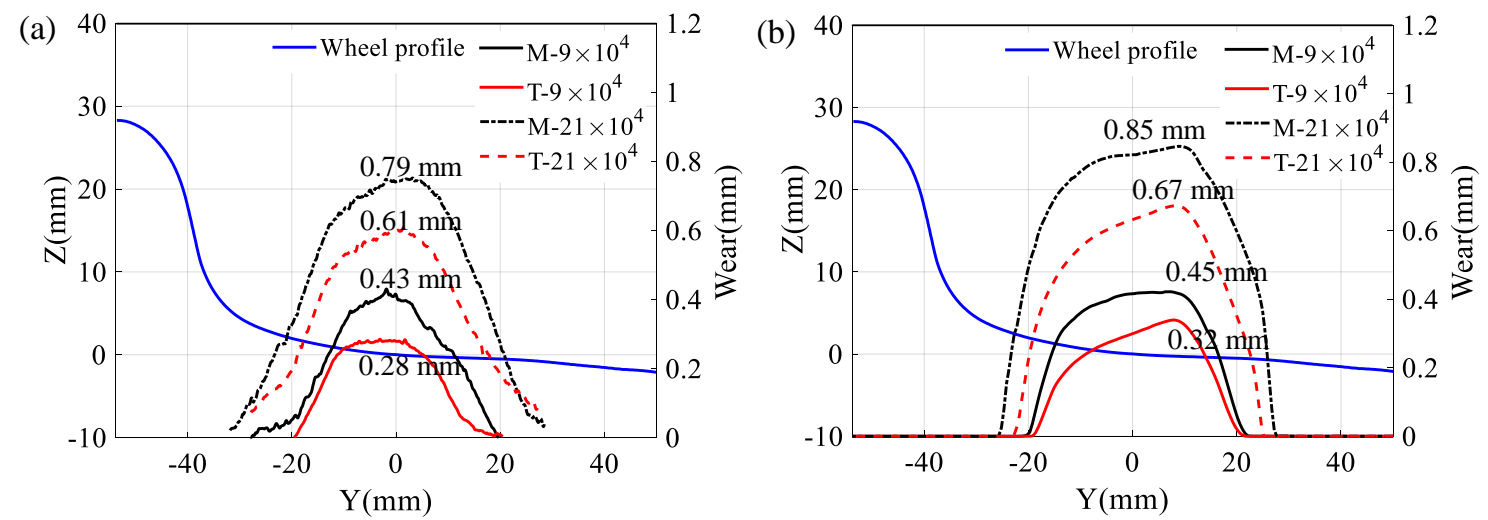

Figure 11 Wheel wear results: (a) field experiments and (b) simulation.
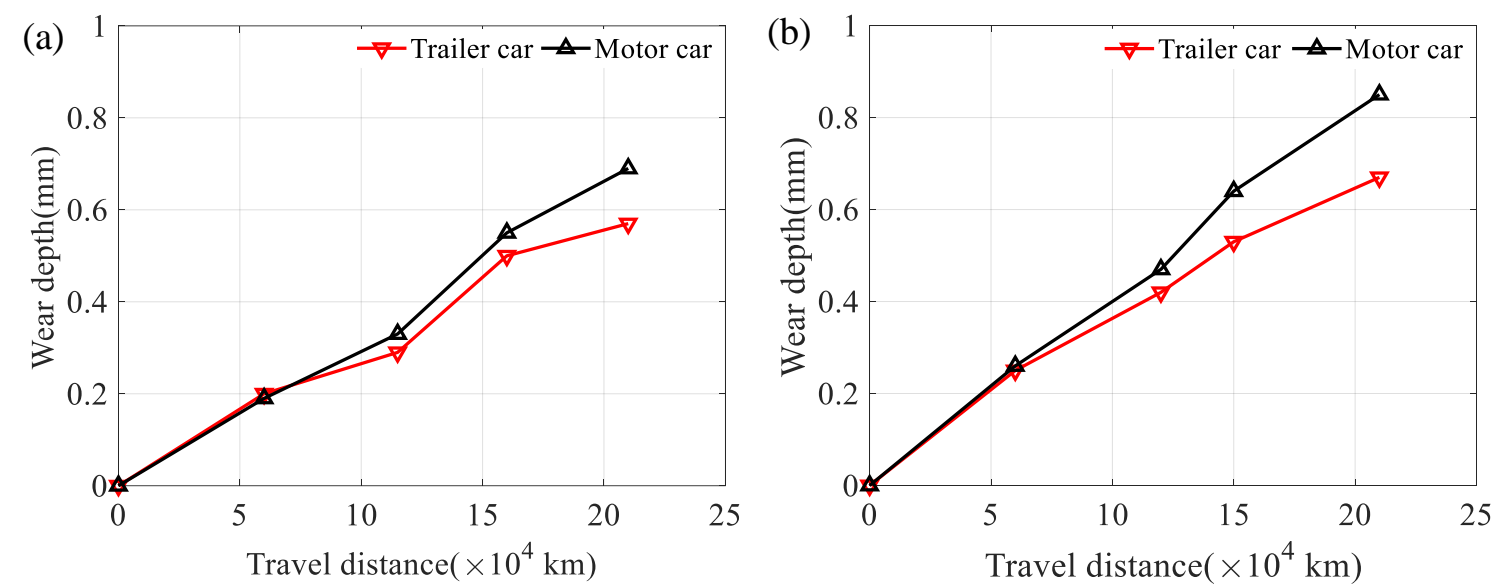

Figure 12 Maximum wheel-wear depths of the motor and travel cars as functions of travel distance: (a) field experimental tests and (b) simulation. 
Figure 12 compares the maximum wheel-wear depths in the motor and unpowered cars obtained in the experimental tests and numerical simulations. The maximum wear depths of both cars were proportional to the travel distance, and the field tests and numerical simulations showed good agreement. The maximum amplitudes of the wheel-wear depth in the motor and unpowered cars nearly coincided over travel distances up to $6 \times 10^{4} \mathrm{~km}$. At longer travel distances, the wheel wear became increasingly deeper in the motor car than in the unpowered car.

\section{Conclusions}

Unlike previous models, the present model considers the detailed coupling effects between the traction transmission system and the motor car of a high-speed train. These effects reflect the power transmission path from the traction motor to the wheelset, and improve the realism of the simulated traction characteristics during the vehicle operation. The model assumes the real track parameters and the track irregularities of a typical high-speed main line in China. The wear was predicted by the Archard wear model and a FASTSIM algorithm, and a specific running distance was assigned in the profile updating procedure. Using the proposed dynamics and wheel-wear models, the wheel wear of the motor and unpowered cars, and their difference, was predicted during a re-profiling cycle. The performance of the wheel-wear characteristics of the motor and unpowered cars were assessed by a suitable evaluation index (the contact patch energy). Moreover, the dynamic performance of the gear transmission system was assessed in terms of its transmission stability during the re-profiling cycle. Finally, to validate the proposed model, the simulation results were compared with the wheel-wear depths measured in long-term field tests of a typical high-speed train in China.

The simulation and field tests confirmed that the motor car experienced more severe wheel wear than the unpowered car. This result was attributed to the higher longitudinal creepage in the motor car, induced by the traction characteristics. At longer distances, the contact patch energy was clearly higher in the motor car than in the unpowered car. The higher contact patch energy and deeper wheel wear reflect the worse wheel-wear status of the motor car when compared to the unpowered car.

Due to continuous wheel wear, the dynamic performance of the gear transmission system (transmission stability and gear interaction) degraded with increasing operation mileage. The standard deviations of the gear mesh force, DTE, gear contact stress and maximum contact stress were $39.8 \%, 31.6 \%, 39.0 \%$ and $10.1 \%$ higher, respectively, after travelling for $21 \times 10^{4} \mathrm{~km}$ than at the start of the simulation.

The proposed model can also assess the dynamic performance of the motor car and traction transmission system (such as vibration, fatigue and damage) in any railway system, especially under traction and/or braking conditions. 


\section{Acknowledgments}

The University of Huddersfield is licensed by Dassault Systemes Deutschland GmbH to use the Simpack software. The first author thanks the support of the China Scholarship Council.

\section{Funding}

The author(s) is grateful for the financial support provided by the National Key Research and Development Program of China (Grant No 2016YFB1200401), State Key Laboratory of Traction Power, Southwest Jiaotong University (No.2016 TPL_T04) and Sichuan Science and Technology Program (2019JDRC0081).

\section{References}

1. W. M. Zhai, Vehicle-track coupling dynamics. Beijing, China: Science Publishing House; 2007. 2. A. Shebani, S. Iwnicki, Prediction of wheel and rail wear under different contact conditions using artificial neural networks, Wear 2018;406-407: 173-184.

3. A. Ramalho, Wear modelling in rail-wheel contact, Wear 2015; 330-331: 524-532.

${ }^{4}$ C. Hardwick, R. Lewis, D. T. Eadie, Wheel and rail wear-Understanding the effects of water and grease, Wear 2014; 314 (1-2): 198-204.

5. R. Enblom, Deterioration mechanisms in the wheel-rail interface with focus on wear prediction: a literature review, Veh Syst Dyn 2009; 47: 661-700.

6. H. Krause, G. Poll, Wear of wheel-rail surfaces, Wear 1986; 113 (1): 103-122.

7. J. F. Archard, Contact and rubbing of flat surfaces, J Appl Phys 1953; 24 (8): 981-988.

8. F. Braghin, R. Lewis, R. Dwyer-Joyce, S. Bruni. A mathematical model to predict railway wheel profile evolution due to wear. Wear, 2006, 261(11-12): 1253-1264.

9 M. Ignesti, M. Malvezzi, L. Marini, E. Meli, A. Rindi. Development of a wear model for the prediction of wheel and rail profile evolution in railway systems. Wear, 2012, 284: 1-17.

10 M. Ignesti, A. Innocenti, L. Marini, E. Meli, A. Rindi. Development of a model for the simultaneous analysis of wheel and rail wear in railway systems. Multibody System Dynamics, 2014,31 (2), 191-240.

11 A. Innocenti, L. Marini, E. Meli, G. Pallini, A. Rindi. Development of a wear model for the analysis of complex railway networks. Wear, 2014, 309(1-2): 174-191.

12. R. Enblom, M. Berg, Impact of non-elliptic contact modelling in wheel wear simulation, Wear 2008; 265 (9-10): 1532-1541.

13. T. Jendel, M. Berg, Prediction of wheel profile wear - Methodology and verification, Veh Sys Dyn 2002; 37 (s1): 502-513.

14. T. Jendel, Prediction of wheel profile wear-comparisons with field measurements, Wear 2002; 253 (1-2): 89-99.

15. J. De Arizon, O. Verlinden, P. Dehombreux, Prediction of wheel wear in urban Railway transport: comparison of existing models, Veh Syst Dyn 2007; 45: 849-866.

16. G. Q. Tao, Z. F. Wen, X. Zhao, X.S. Jin. Effects of wheel-rail contact modelling on wheel wear 
simulation, Wear 2016; 366-367: 146-156.

17 J. Pombo, J. Ambrosio, M. Pereira, R. Lewis, R. Dwyer-Joyce, C. Ariaudo, N. Kuka. Development of a wear prediction tool for steel railway wheels using three alternative wear functions. Wear, 2011, 271(1-2): 238-245.

18. A. Bevan, P. Molyneux-Berry, B. Eickhoff, M. Burstow. Development and validation of a wheel wear and rolling contact fatigue damage model, Wear 2013; 307 (1-2): 100-111.

19. H. Kazuyuki, M. Fumiko. Influence of wheel/rail tangential traction force on thermal cracking of railway wheels, Wear 2012; 289 (25): 112-118.

20. Z. W. Wang, W. H. Zhang, Z. H. Yin, Y. Cheng, G.H. Huang. Effect of vehicle vibration environment of high-speed train on dynamic performance of axle box bearing, Veh Syst Dyn 2019; 57 (4): 543-563.

21. G.H Huang, N. Zhou and W. H. Zhang. Effect of internal dynamic excitation of the traction system on the dynamic behavior of a high-speed train. Proc IMechE, Part F: J Rail and Rapid Transit 2016; 230:1899-1907.

22. Z. W. Wang, G. M. Mei, W. H. Zhang, Y. Cheng, H.Y. Zou, G.H. Huang, F.Z. Li. Effects of polygonal wear of wheels on the dynamic performance of the gearbox housing of a high-speed train. Proc IMechE, Part F: J Rail and Rapid Transit 2018; 232 (6): 1852-1863.

23. Z.G. Chen, W. M. Zhai and K. Y. Zhai. Locomotive dynamic performance under traction/braking conditions considering effect of gear transmissions. Veh Syst Dyn 2018; 56 (7): 1-21.

24 J. Pombo, J. Ambrósio, M. Pereira, R. Lewis, R. Dwyer-Joyce, C. Ariaudo, N. Kuka. A study on wear evaluation of railway wheels based on multibody dynamics and wear computation. Multibody System Dynamics, 2010, 24(3): 347-366.

25. J. W. Zhang, B. Xu, X. Q. Guan. A combined simulation procedure for wear assessment of the HXN5 locomotive, Wear 2014; 314 (1-2): 305-313.

26 J. Pombo, J. Ambrósio, M. Pereira, R. Lewis, R. Dwyer-Joyce, C. Ariaudo, N. Kuka. A railway wheel wear prediction tool based on a multibody software. Journal of theoretical and applied mechanics, 2010, 48: 751-770.

27. Mauer, L.: Force Element 225 Gear Wheel. Internal report, Intec GmbH, Wessling, Germany 2005.

28. R. Luo, H. L. Shi, W. X. Teng, C.Y. Song. Prediction of wheel profile wear and vehicle dynamics evolution considering stochastic parameters for high-speed train, Wear 2017; 392-393: 126-138.

29. T. G. Pearce, N. D. Sherratt, Prediction of wheel profile wear, Wear 1991; 144 (1-2): 343-351.

30. I. McEwen, R. Harvey, Interpretation of wheel/rail wear number, ReportRef: TM VDY004, British Rail Research, 1986. 